\title{
USING SIMILARITY TRANSFORMATION TECHNIQUE FOR SELECTING THE OPTIMAL FIXED POINTS FOR OVER- CONSTRAINED GEODETIC NETWORKS
}

\author{
Rahil A. M., El Gohary A. M., Doma M. I., El Shony A. F. \\ Civil Engineering Department, Faculty of Engineering, \\ Minoufiya University Shebin El-Kom, Egypt
}

\begin{abstract}
In the case that more constraints than necessary to remove the network datum defects have been defined, a new type of geodetic network will be obtained " over - constrained geodetic network “. Datum changes may be obtained by Similarity Transformations in order to select the proper coordinate datum for geodetic networks as well as to analyse the stability of the reference points. The purpose of this research paper is to choose the optimal fixed points for over - constrained geodetic networks by using Similarity Transformation. A numerical example is presented.

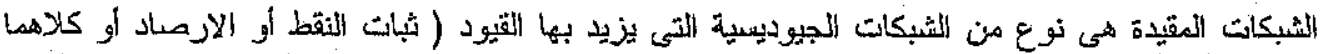

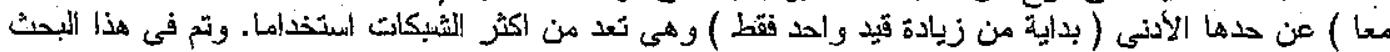

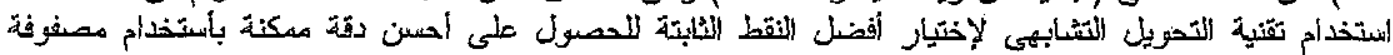

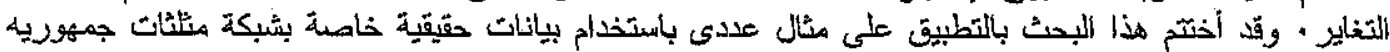





\section{INTRODUCTION}

One of the most important types of the geodetic networks is over - constrained geodetic network which has more constraints than necessary to remove the network datum defects. These network constraints provide information to the adjustment process about the absolute position and orientation of the network.

The main objective of this paper is to introduce the using of Similarity Transformation method to choose the optimal fixed points for over - constrained geodetic network and to analyse the stability of these fixed points. In addition the use of Similarity Transformation to transform the covariance matrix from one computational base to another one is introduced and discussed.

A numerical example respresenting a part of egyptian geodetic network were given in order to illustrate the feasibility of the given procedure.

The results shows the efficieny of the proposed procedures.

The main types of geodetic network can be classified as follows: [US Army Corps, 2002]

1) Free networks, of which the all coordinate of its points still need to be calculated.

2) Minimum constraints networks, of which contains the ordinary minimum constraints for adjustment and other parameters are free.
3) Over constrained networks, of which contains more constraints than necessary to remove the network datum defects have been defined and there are points still need to be determined.

4) Fully constrained networks: In a fully constrained adjustment all stations in the reference network are assumed to have well-known coordinates (i.e., stable points), and these are fixed with zero error in the adjustment process. With fully constrained adjustments, for the monitoring networks, only the monitoring point stations are allowed to float and adjust in position.

\section{OVER - CONSTRAINED NETWORK}

In the case that more constraints than necessary to remove the rietwork datum defects have been defined; for instance, fixing two or more stations for a two-dimensional trilateration network, a new type of geodetic network will be obtained "over-constrained geodetic network".

Assume the number of constraints defined and the number of network datum defects are $n_{c}$ and $n_{d}$ respectively. For the over - constrained network case,we have : [Kuiang, 1996]

$$
n_{c}>n_{d}
$$

Depending on whether a network is established in one-, two-, or three-dimensional space and the geodetic measurements made within the network, the 
type and total number of datum parameters for different types of geodetic networks were given in Table (1). [ Doma, 2008 ].

Table (1) Datum parameters of geodetic networks

\begin{tabular}{|c|c|c|c|c|}
\hline \multicolumn{2}{|c|}{ Network Type } & \multicolumn{2}{|c|}{ Datum Patameters } & No. \\
\hline \multirow{3}{*}{\multicolumn{2}{|c|}{$\begin{array}{c}\text { Three-Dimensional } \\
\text { Net. }\end{array}$}} & 3.tronslations & $\begin{array}{l}t_{x}=\text { translation in } x \text {-dir. } \\
t_{y}=\text { translation in } y \text {-dir. } \\
t_{z}=\text { translation in } z \text {-dir. }\end{array}$ & \multirow{3}{*}{7} \\
\hline & & 3.rotations & $\begin{array}{l}\omega_{\mathrm{x}}=\text { rotation about } \mathrm{x} \text {-dir. } \\
\omega_{\mathrm{y}}=\text { rotation about } \mathrm{y} \text {-dir. } \\
\omega_{\mathrm{z}}=\text { rotation about } \mathrm{z} \text {-dir. }\end{array}$ & \\
\hline & & 1.scale & $s=$ scale & \\
\hline \multirow{5}{*}{$\begin{array}{l}\text { Horizoutal } \\
\text { Network }\end{array}$} & \multirow{3}{*}{$\begin{array}{l}\text { Trinugulatio } \\
\text { a Nenwoik. }\end{array}$} & 2.translations & $\left\{\begin{array}{l}t_{x}=\text { translation in } x \text {-dir. } \\
t_{y}=\text { translation in } y \text {-dir. }\end{array}\right.$ & \multirow{3}{*}{4} \\
\hline & & 1.rotations & $\omega_{z}=$ rotation ahout $z$-dir. & \\
\hline & & 1.scale & $\mathrm{s}=$ scale & \\
\hline & \multirow{2}{*}{$\begin{array}{l}\text { Trilateratio } \\
\text { n Netwyork }\end{array}$} & 2.transtations & $\begin{array}{l}t_{x}=\text { translation in } x \text {-dir. } \\
t_{y}=\text { translation in } y \text {-dir. }\end{array}$ & \multirow[t]{2}{*}{3} \\
\hline & & 1.rotations & $\omega_{z}=$ rotation about $z-d i r$ & \\
\hline \multicolumn{2}{|c|}{ Vertical Network } & 1.translations & $t_{\mathrm{z}}=$ franslation in z-dir. & 1 \\
\hline
\end{tabular}

In general, let us assume that the constraint equation is described by :

$$
R_{n_{\varepsilon} \times u} \cdot X_{u \times 1}=C_{n_{o} \times 1}
$$

where:

$X$ : the unkown coordinate vector,

$C$ : is a constant vector,

$R$ : is a reliability factor.

with dimensions :

$u$ : number of unkowns,

$\mathrm{n}_{\mathrm{c}}:$ number of constraints defined.

\section{SIMILARITY TRANSFORMATION}

Local geodetic datums have been developed in the past, in order to satisfy the surveying and mapping requirements of countries all over the earth. The broad use of GPS observations, as part of the surveying routine, has shifted the interest from the local to the world geodetic systems. The transformation of coordinates between geodetic systems has always been of interest, but the new needs have made it more important.
The choice of the most appropriate network transformation model is influenced by :

1- The extents of the area for which it is to be applied.

2- The presence of distortion in either of the reference system.

3- The dimension of the reference system (two or three dimenisional), and

4- The accuracy requirements.

The scale factor of such a transformation depends on the orientation but not on the position within the net. A transformation in which the scale factor is the same in all directions is called a Conformal or Similarity Transformation (known also as a Helmert). In case of scale factor is unity the Similarity Transformation is called as an Orthogonal Transformation.

Similarity Transformation were first described in published form by Baarda (1973).

The Similarity Transformation is one of the most commonly used transformation methods due to:

1- The small number of parameters involved.

2- The simplicity of the model which is more easily implemented into software, and

3- The fact that it is adequate for relating two coordinate systems which are homogeneous (no local distortion in scale or orientation).

One disadvantage of the Similarity Transformation method is that both networks are assumed to have only linear distortions (excluding shear components). Often older terrestrial networks do have non-linear distortions because of the adjustment and survey methodologies employed.

Similarity Transformation can be used to select the proper coordinate datum for geodetic networks as well as to analyse the stability of the reference points.

The estimated point coordinates and their covariance matrix can be transformed from one computational base to another without repeating the adjustment process via an Similarity Transformation. The transformation from an arbitrary datum (i) to a certain datum (j) can be achieved for the datum dependent coordinates and their covariance matrix with the following formulas : [ Rahil, 2001]

$$
\begin{aligned}
& x_{j}=S_{j} x_{i} \\
& \left(C_{x}\right)_{j}=S_{j}\left(C_{x}\right)_{i} S_{j}^{T}
\end{aligned}
$$

In which:

$$
S_{j}=S_{m}=1-G\left(G^{T} G\right)^{-1} G^{T}
$$

where:

$\left(C_{x}\right)_{j}$ is the variance - covariance matrix calculated under network datum $j$. 
$\left(C_{X}\right)_{I}$ is the variance - covariance matrix calculated under network datum $i$.

$\mathrm{S}_{\mathrm{m}} \quad$ is the Similarity Transformation matrix, it is idempotent $: j=j^{2}$ and $j=j^{T_{3}}$ hence $j$ is an orthogonal projector.

I is the identity matrix.

$G$ is the transformation matrix.

Often not all points enter the definition of the network datum. Let $P_{X}$ be a diagonal matrix with " 1 " for point that enter the datum definition and " 0 " for all others. So equation (5) will be : [Even,2000]

$$
S=I-G\left(G^{T} \dot{P}_{X} G\right)^{-1} G^{T} P_{X}
$$

The project $G\left(G^{T} P_{X} G\right)^{-1} G^{T} P_{X}$ is no longer square matrix and hence not orthogonal.

Similarity Transformation matrix $\left(\mathrm{S}_{\mathrm{m}}\right)$ is dependent upon:

- Number and geometry of the datum - points contributes to the datum definition.

- The inner constraints matrix ( $G$ ).

- The number of datum defect (d).

\section{APPLIED STUDY AND RESULTS}

In this paper, applications of using Similarity Transformation method to select the proper coordinate datum for over - constrained geodetic networks are performed using real terrestrial data.

first, the data used in this study is explained then, the steps of the solution is summarized finally, the obtained results of computations will be discussed and analysed.

\subsection{USED DATA}

The used data is a part of the Egyptian first order horizontal terrestrial geodetic network. The data consists of six points. These points are well distributed over the Egyptian territory as shown in Figure (1).

These points $(2-D)$ cartesian coordinates are defined relative to WGS 84 (World Geodetic System 1984 ) datum. The used data has been collected from El Habiby. [ El Habiby, 2002 ].

\subsection{THE SOLUTION STEPS OF CASE STUDY}

The solution steps for the proposed approach will be given below :

\section{Given:}

1- Approximate coordinates for used points,

2- The observational plan for the geodetic network (trilateration, triangulation or hybrid network),

3- The fixed points of the geodetic network,

4- The achievable accuracy of the used EDM instrument.



Figure (1): The Location of the Selected Points

\section{Required:}

The optimal fixed points for this over-constrained geodetic network.

\section{* The solution stens of the case-study can} be summarized as follows:

a) Selecting the intial fixed points of the network:

A computer program using the PC - Matlab version is used. This program automatically selects any two points to be the intial fixed points of the network,

b) Computation of design matrix (A) for this case:

This case can be achieved, by using the given coordinates of the existing points, with the selected fixed points to evaluate the partial derivatives within the matrix (A),

c) Computation of an actual weight matrix (P) for this case:

In this paper, we assume that accuracies of the horizontal components of the measurements vectors $(X, Y)$ and the accuracy of the vertical component $(Z)$ are equal,

d) Calculation Variance - Covariance matrix $\left(C_{X}\right)_{i}$ of the over-constrained network:

The Variance - Covariance matrix of the astimated coordinates can be computed by :

$$
\left(C_{X}\right)_{j}=\sigma_{o}^{2}\left(A^{T} P A\right)^{-1}
$$

$$
\text { where: }
$$

$\left(\sigma_{0}\right)^{2}$ is the variance of unit weight or variance factor, 
e) Computation of the Similarity Transformation matrix $\left(\mathrm{S}_{\mathrm{j}}\right)$ :

The Similarity Transformation matrix can be computed by:

$$
S_{j}=1-G\left(G^{T} G\right)^{-1} G^{T}
$$

f) Computation of Variance - Covariance matrix ( $C_{\underline{X}}$ Li under network datum (j):

The Variance - Covariance matrix $\left(\mathrm{C}_{\mathrm{X}}\right)_{\mathrm{j}}$ can be computed by using Similarity Transformation matrix as shown below:

$$
\left(C_{x}\right)_{j}=S_{j}\left(C_{x}\right)_{j} S_{j}^{T}
$$

The standard deviation vectors $\left(\sigma_{\mathrm{X}}, \sigma_{\mathrm{Y}}\right)$ can be obtained from the Variance - Covariance, which has been computed by using Similarity Transformation matrix, as shown below :

$\left(C_{x}\right)_{j}=\left[\begin{array}{ccccc}\sigma_{X 1}^{2} & \sigma_{X 1 Y 1} & \ldots & \sigma_{X 1 X n} & \sigma_{X 1 Y n} \\ \sigma_{X 1 Y 1} & \sigma_{Y 1}^{2} & \ldots & \sigma_{X n Y 1} & \sigma_{Y n Y 1} \\ \ldots & \ldots & \ldots & \ldots & \cdots \\ \sigma_{X 1 X n} & \sigma_{X n Y 1} & \ldots & \sigma_{X n}^{2} & \sigma_{X n Y n} \\ \sigma_{X 1 Y n} & \sigma_{Y I Y 1} & \ldots & \sigma_{X n Y n} & \sigma_{Y n}^{2}\end{array}\right]$

where :

$\sigma_{X}$ is the standard deviation in X-axis.

$\sigma_{Y}$ is the standard deviation in $Y$-axis.

g) Reselecting another fixed points:

The program automatically selects another two points.

h) Recomputation of Variance - Covariance matrix $\left(\mathrm{C}_{X}\right)_{\mathrm{j}}:$

This can be achieved by, resolving equations from (b) to (f).

i) The selection of the case of the optimal fixed points:

The case of the optimal fixed points is the case which has the minimum values of the maximum values of standard deviation vectors which are obtained from Variance - Covariance matrices of different cases of fixed points. This selection is automatically performed by the program.

After the selection of the optimal fixed points, the program gives the Variance - Covariance matrix and the Figure of this case of the optimal fixed points.

\subsection{APPLICATION OF THE PROPOSED ALGORITHM}

Let us assume that, the Egyptian trilateration geodetic network, (as shown in Figure (1), used in this paper, has been measured using EDM instrument with achievable accuracy

$$
\sigma_{s}^{2}=(0.5 \mathrm{ppm} . \mathrm{S})^{2}
$$

where $S$ is the distance computed from the approximate coordinates.

\section{DISCUSSION OF THE OBTAINED RESULTS}

We used the standard deviation obtained from Variance - Covariance matrix as a precision criteria for this example.

Table ( 2 ) includes the precison of the network using Similarity Transformation technique. This results shows that.

a) The standard deviation of the points are rauged from $(193.25) \mathrm{mm}$ at point $\left(\mathrm{P}_{2}\right)$ in case $(3)$ to (474.72) $\mathrm{mm}$ at point $\left(P_{1}\right)$ in case $(12)$.

b) The optimal fixed points are points $\left(\mathbf{P}_{1}, \mathbf{P}_{\mathbf{4}}\right)$, these optimal fixed points have been selected automatically by using Similarity Transformation technique.

c) The Variance - Covariance matrix of case ( 3 ) is shown at equation (10).

d) The final Figure of case ( 3 ) is shown in Figure (2).

$$
\left(C_{x}\right)_{j}=\left[\begin{array}{cccccccc}
0.038 & -0.017 & -0.013 & 0.011 & -0.019 & -0.001 & -0.006 & 0.007 \\
-0.017 & 0.025 & 0.006 & -0.011 & 0.006 & -0.006 & 0.004 & -0.007 \\
-0.013 & 0.006 & 0.014 & -0.007 & 0.001 & -0.001 & -0.002 & 0.002 \\
0.011 & -0.011 & -0.007 & 0.029 & -0.015 & -0.015 & 0.010 & -0.003 \\
-0.019 & 0.006 & 0.001 & -0.015 & 0.030 & 0.013 & -0.011 & -0.004 \\
-0.001 & -0.006 & -0.001 & -0.015 & 0.013 & 0.024 & -0.010 & -0.003 \\
-0.006 & 0.004 & -0.002 & 0.010 & -0.011 & -0.010 & 0.020 & -0.005 \\
0.007 & -0.007 & 0.002 & -0.003 & -0.004 & -0.003 & -0.005 & 0.013
\end{array}\right]
$$


A. Rahil, A. El Gohary, M. Doma, A. El Shony, "Using Similarity Transformation Technique for Selecting ... "

Table (2) The precison of the network using Similarity Transformation technique for different cases

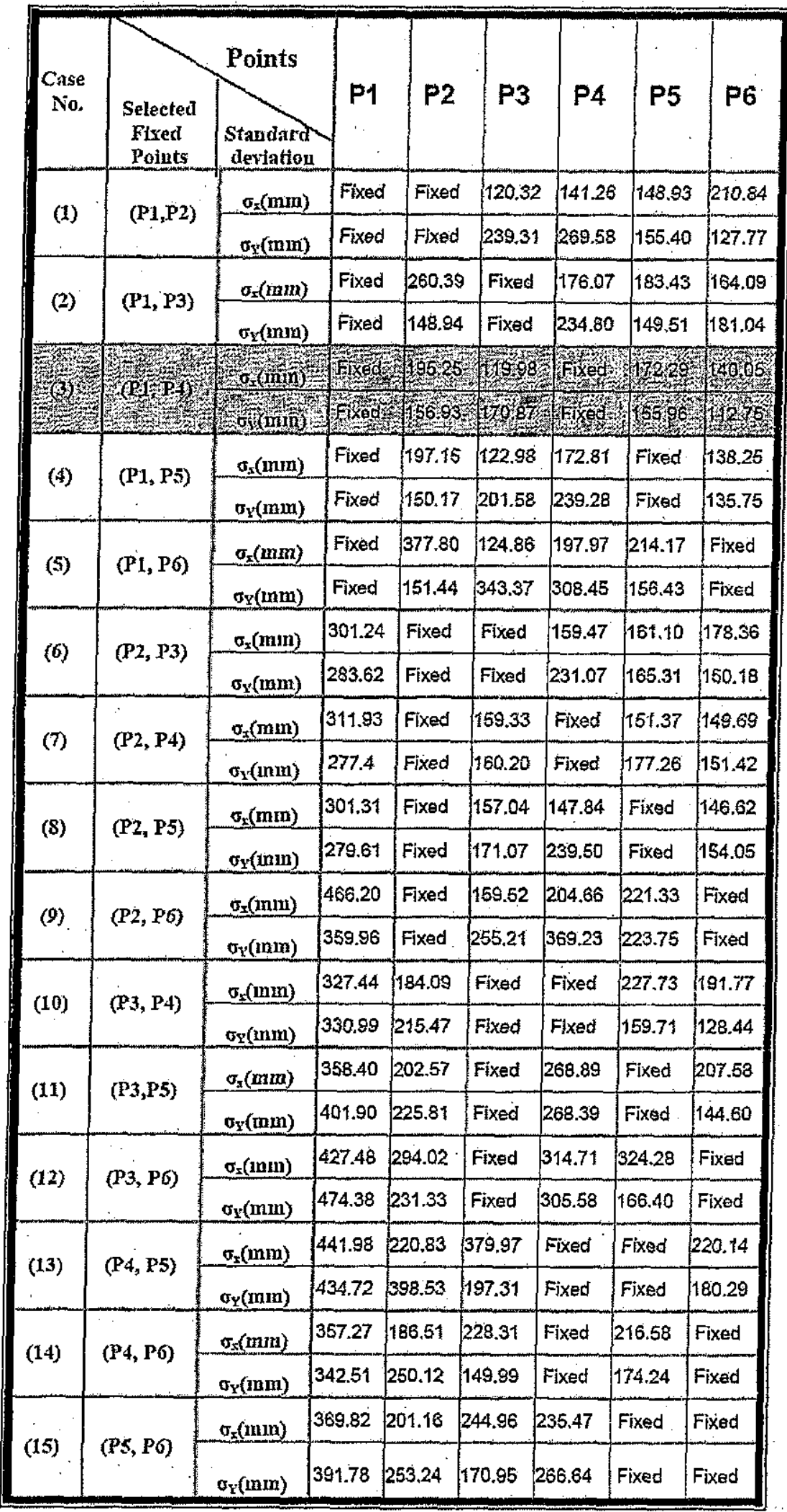

The optimal fixed points case. 


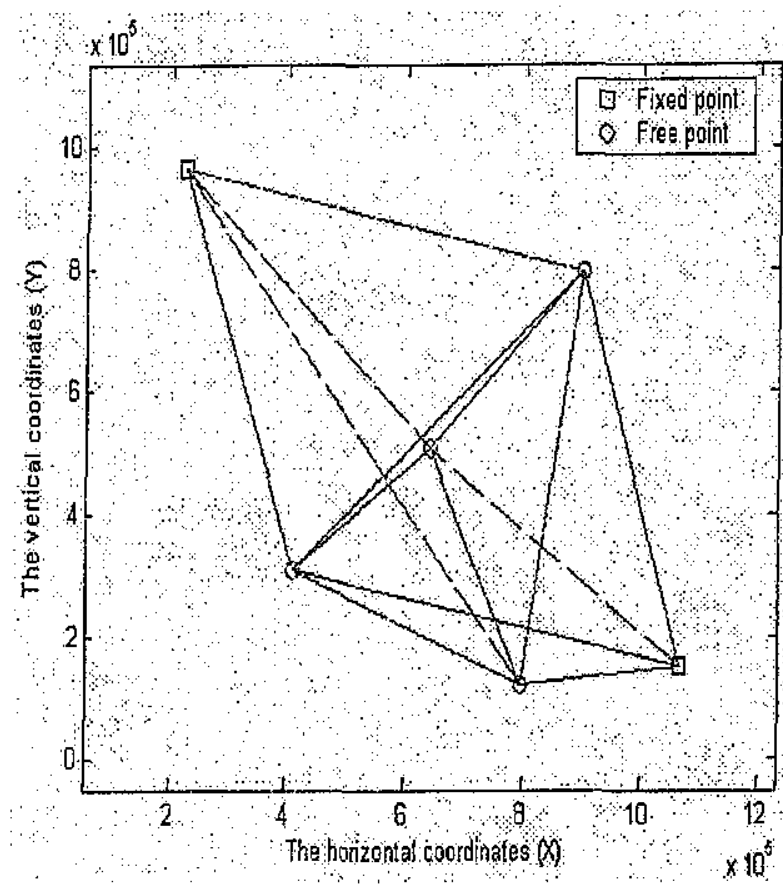

Figure (2): The Final Figure of the case of Optimal Fixed Points (Case (3))

\section{CONCLUDING REMARKS}

Depending on the obtained results and the analysis that carried out on it, we can summarize the following conclusions:

* Similarity Transformation is a powerful computational tool which is capable of transforming one adjustment to another without going through the procedure of every adjustment.

* We can easily use the Similarity Transformation method to select the proper coordinate datum for the over - constrained geodetic network.

* From the present paper, one can select the optimal fixed points automatically without using the trial and error method.

\section{REFRENCES}

Baarada W. (1973). "S-Trasformations and Criterion matrices." Neth. Geod. Com., publ. on Geodesy, New Series 5, no. 1, Deift, Netherlands.

Doma M.l. (2008). The optimal improvement of geodetic network using interactive graphics system, Doctoral thesis at the faculty of Engineering, Minoufiya University, Cairo, Egypt.

El-Habiby M.M. (2002). Effects of coordinate transformation from Helmert 1906 old system to WGS84 GPS new system on geodetic networks and related mapping system in Egypt, M. Sc. Thesis at the Public Works Department, Faculty of Engineering, Ain Shams University, Cairo, Egypt.

Even, G. and Tzur (2000). Datum Definition for GPS Network, Aalborg University, Denmark. Survey review, vol. 35 , no. 277 , pp. $475-485$.

Kuang, S.L. (1996)."Geodetic Network Analysis And Optimal Design: Concept And Applications." Ann Arbor Press, Inc., Chelsea, Michigan.

Rahil, A.M. and Abo-halima, H. (2001). "Application of Similarity Transformation in geodetic monitoring networks" ERB, Vol. 24, No. 4, 2001, Menufiya University, Faculty of Eng., Shebien El-Koom, Egypt, ISSN $1110-1180$.

US Army Corps of Engineering (2002). "Structural Deformation Surveying EM 1110-2-1009", Department of the Army, Washington, June 2002. 